Running Head: Pictures of tools with matched objects and non-objects

\title{
A validated set of tool pictures with matched objects and non-objects for laterality research
}

\author{
Ark Verma $^{1}$ and Marc Brysbaert ${ }^{2}$ \\ Department of Experimental Psychology ${ }^{1,2}$, \\ Faculty of Psychological and Educational Sciences, \\ University of Gent, \\ Belgium.
}

Corresponding Author: Ark Verma

Department of Experimental Psychology,

Faculty of Psychological and Educational Sciences,

University of Gent,

Henri Dunantlaan-2,

9000-Gent,

Belgium.

E-mail: ark.verma@ugent.be

Phone: +32-489201373 


\begin{abstract}
Neuropsychological and neuroimaging research has established that knowledge related to tool use and tool recognition is lateralized to the left cerebral hemisphere. Recently, behavioral studies with the visual half field technique have confirmed the lateralization (Verma \& Brysbaert, 2011; Garcea, Almeida \& Mahon, 2012). A limitation of this research was that different sets of stimuli had to be used for the comparison of tools to other objects, and objects to non-objects. Therefore, we developed a new set of stimuli containing matched triplets of tools, other objects, and non-objects. With the new stimulus set we successfully replicated the findings of no visual field advantage for objects in an object recognition task, combined with a significant right visual field advantage for tools in a tool recognition task. The set of stimuli is available as supplementary materials to this article.
\end{abstract}




\section{A validated set of tool pictures with matched objects and non-objects for laterality research}

Manufacturing and using tools have been considered important milestones in the evolution of the human brain and date back at least 2.5 million years (Ambrose, 2001). Although tool use has been considered as a typically human skill (Oakley, 1956), there is evidence that many species ranging from birds (Lefebvre, Nicolakakis, \& Boire, 2002) to elephants (Hart, Hart, McCoy \& Sarath, 2001), crows (Hunt, 1996), orangutans (van Schaik, Ancrenaz, Borgen Galdikas, Knott, Singleton, Suzuki, Utami \& Merill, 2003), capuchin monkeys (Westergaard and Fragaszy, 1987), dolphins (Krutzen, Mann, Heithaus, Connor, Beider \& Sherwin, 2005) and chimpanzees (Boesch and Boesch, 1990) engage in various forms of tool use.

Several definitions have been offered to differentiate "tools" from other objects and "tool use" from other types of behavior. For example: an early definition of tool use offered by Jane Goodall describes tool use as, "the use of an external object as a functional extension of the mouth or beak, hand or claw, in the attainment of an immediate goal" (van Lawick-Goodall, 1970, page 195). Alcock (1972, page 464) defines tool use as "the manipulation of an inanimate object, not internally manufactured, with the effect of improving an animal's efficiency in altering the form or position of some separate object". Finally, Beck (1980, page 10) defines tool use as "the external 
employment of an unattached environmental object to alter more efficiently the form, position or condition of another object, another organism, or the user itself when the user holds or carries the tool during or just prior to use and is responsible for the proper and effective orientation of the tool". However, Preston (1998) observes that various definitions of tool use basically attempt to formalize for scientific purposes a "folk category" of tool use which underwrites the definitions of "tools" found in dictionaries, and hence are simply 'inadequate'. Indeed, many examples of animal and human behavior can be cited that may not be covered within these definitions.

To paraphrase the various possible definitions of tools and tool use that differ in their scope and usefulness (read Preston, (1998) \& Amant \& Horton, (2008) for a detailed discussion), a "tool" has to be an "external", "inanimate" object employed by a user for a "goal-directed" action. For the purpose of the current study we followed a definition of tools proposed by Frey (2007), who defines "tools as manipulable objects that are used to transform an actor's motor output into predictable mechanical actions in order to attain specific goals". Accordingly, all objects presented as tools in our study were man-made, hand manipulable and had typical and well-established uses. For example: a hammer is typically associated with driving nails. Hence, in the current study the tools are defined in "typical" contexts so that we can interpret the consequent findings in a constrained frame of reference and avoid ambiguity.

Even though a variety of species have been reported to be engaged in tool use, the understanding and use of tools in humans goes far beyond the animal skills. Evidence 
indicates that humans possess specialized neuronal mechanisms allowing them to understand the functional properties of tools, both simple and complex (Frey, 2007). Not only does the human brain have dedicated regions for tool use, these regions are lateralized to the left hemisphere, which is also the dominant hemisphere for language and related functions (Lewis, 2006, Frey 2004). Indeed, a lot of evidence from clinical studies indicates a major role of the left hemisphere of the brain in accessing and processing the knowledge of tools and tool use. For instance, Hermsdorfer, Li, Randerath, Goldenberg \& Johanssen (2012) found that patients with left hemisphere stroke exhibited reduced hand rotation at the bowl and the plate in pantomiming as well as actual use. Randerath, Goldenberg, Spijkers, Li \& Hermsdorfer (2010) found a large area of lesion overlap in the left supramarginal gyrus for patients with impaired tool use whereas lesion overlap in the left inferior frontal gyrus and left angular gyrus for patients who were impaired in tool grasping. Also, Goldenberg \& Spatt (2009) observed that parietal lesions involving the left supramarginal gyrus impaired tool use (both common and new) and left frontal lesions affected tool use and tool knowledge. Further, Osiurak et al. (2009) found that left brain damaged patients had more difficulties on the unusual use of objects when compared to healthy controls or right brain damaged patients. Finally, Goldenberg, Hermsdorfer, Glindemann, Rorden, and Karnath (2007) examined 44 patients with left sided cerebrovascular accidents, and found that lesions in the inferior frontal gyrus and adjacent portions of the insula and precentral gyrus led to defective pantomiming ability. 
Adding to the evidence from clinical studies, large number of neuroimaging studies also demonstrate greater involvement of left hemisphere areas in tasks related to tool knowledge and use.

Typically, in neuroimaging research, participants are asked to pantomime specific tool operations, and the brain activity related to these movements is compared with that of repetitive limb movements (Choi, Na, Kang, Lee, Lee \& Na, 2001), meaningful hand gestures (Fridman et al. 2006) or meaningless hand movements (Fridman, Immisch, Hanakawa, Bohlhalter, Waldvogel, Kansaku, Wheaton, Wu, and Hallett, 2006, Grezes \& Decety, 2001). Other researchers present participants with pictures of tools vs. pictures of humans, animals, houses, faces, or even scrambled images, and measure the differences in brain activity (Beauchamp, Haxby \& Martin, 2002; Chao, Haxby \& Martin, 1999; Chao \& Martin, 2000). It has been suggested that for such comparisons it is best to compare tools to other man-made objects, such as houses, because otherwise it is difficult to be sure that the observed differences in brain activity are specific to tool use or could be due to other categorical differences, such as that between man-made objects and natural, animate organisms (Kallebach, Brett, \& Patterson, 2003).

Using a variety of tasks and paradigms, neuroimaging research has uncovered a range of left hemispheric cortical areas important for tool knowledge and tool-use behavior. For instance, the left ventral pre-central gyrus in the frontal lobe (ventral Premotor cortex, VPMCx), the left Intraparietal sulcus (IPS) in the posterior parietal cortex, and the posterior middle temporal gyrus (PMTG) either in the left hemisphere 
alone or bilaterally (Beauchamp et al. 2002; Chao \& Martin, 2000; Perani, Schnur, Tettamanti, Gorno Tempini, Cappa, and Fazio,. 1999) have been linked to toolidentification. Johnson-Frey, Newman-Norlund \& Grafton (2005) reported that sites in the left inferior frontal, inferior parietal and posterior temporal cortices are involved in planning tool use regardless of the hand used. Finally, Kroliczak \& Frey (2009) provided evidence that the left intraparietal sulcus, supramarginal gyrus, caudal superior parietal lobule and dorsal pre-motor cortices, are engaged in planning both transitive and intransitive actions.

While multiple accounts implicate the left hemisphere superiority in tool- related behavior, some evidence for right hemisphere contribution in tool processing has also been reported (Frey, 2008). Specifically, Hamilton \& Grafton (2008) reported that right inferior parietal and right inferior frontal cortices area encode physical outcomes of actions in the real world. In a similar vein, Hartmann, Goldenberg, Daumuller \& Hermsdorfer (2005) reported that right brain damaged patients had problems in keeping track of multiple step action sequences, which might be vital for tool-use behavior. Further, while Frey (2008) demonstrates activations in the left posterior parietal (pPar), dorsal and ventral premotor cortices along with middle frontal gyrus and frontal and posterior temporal cortices during tool use pantomimes; he also reports relatively smaller activations in the homologous right hemisphere sites. Also, left handed patients have been found to show signs of apraxia following right hemisphere lesions also (Valenstein \& Heilman, 1979; Dobatao, Baron, Barriga, Pareja, Vela \& Sanchez Del Rio, 2001). 
Finally, left handed participants have also been reported to show greater recruitment of right parietal, frontal and temporal cortices than right handed participants while listening to the sounds made by hand-held tools versus the sounds made by animals, reflecting a possible automatic activation of praxis representations lateralized to the right hemisphere (Lewis, 2006).

Together, findings from clinical and neuroimaging research indicate that knowledge and skills related to tools, are represented in functionally specialized networks distributed majorly but not exclusively over the left hemisphere (Frey, 2004). Frey (2004) postulates two major networks i.e. the conceptual network that represents tool knowledge and consists of areas that display activation during semantic tasks and the skill network which consists of areas activated during retrieval of tool related skills. Major areas represented in the conceptual network include the left and right fusiform gyri, the left middle temporal and superior temporal gyrus, the left ventral premotor cortices, Brodmann's Areas 44/45 (Broca's area) and the left medial Frontal Gyrus. The conceptual network mediates tool observation, tool naming, action word generation and observing action goals. The skill network comprises of Brodmann's Areas 7, 39, 40, left medial and anterior inferior parietal sulcii, left dorsal premotor cortices and left medial frontal gyrus. The skill network accomplishes skill representation/retrieval, reaching, grasping and manipulation related activities (for a detailed description of the two networks, see Frey 2004). Tool-use behavior is accomplished by coordinated functioning 
of the smaller modules of these two networks, which carry out specific component functions (Frey, 2004).

To examine whether the lateralization of tool-use skills is affected by handedness, Frey, Funnell, Gerry and Gazzaniga (2005) examined the relationship between hand dominance and tool use skills by comparing a right-handed male and a left-handed female callosotomy patient and found a left hemisphere advantage for pantomiming actions associated with familiar tool-objects and pictures in both patients. Later, Frey (2008) concluded that tool-use behavior in majority of humans is lateralized to the left hemisphere, regardless of handedness.

Although tool-use lateralization appears to be unrelated to handedness, tool-use related behavior appears to be co-lateralized along with the linguistic capabilities of individuals. Recently, Vingerhoets, Alderweireldt, Vandemaele, Cai, Van der Haegen, Brysbaert, and Achten (2013) compared a group of typically lateralized individuals with left hemisphere speech dominance, to a group of atypically lateralized individuals with right hemisphere speech dominance (as assessed with fMRI) and found that the brain areas involved in tool pantomiming were lateralized to the same hemisphere as language production, regardless of handedness. Additional proof for the same was provided by Uomini \& Meyer (2013), who demonstrated using fTCD that acheulean stone tool production and cued word generation caused almost identical cerebral blood flow lateralization in their participants; and concluded that stone tool making and language are served by common neural substrates. 
Co-lateralization of tool-making or tool-use along with the linguistic capabilities of humans has been viewed as a significant clue to the co-evolution of both these behaviors in humans (Frey, 2008). Several authors (Corballis, 2009; Corballis, 2010; Corballis, Badzakova-Trajkov \& Haberling, 2012; Stout \& Chaminade, 2012) have explored hypotheses that propose that language evolved as a result of advances in manual praxis. More recently, it has been proposed that the human brain is endowed with a system of mirror neurons for observing and matching actions, which might be homologous to the macaque mirror neuron system (Rizollatti, 2005; Rizzollatti, Fogasi \& Gallese, 2002). Further, it has been argued that this mirror system has aided the evolution of manual gestures (for e.g. grasping) into meaningful communicative symbols (akin to modern day sign-language) and later into full fledged language articulated using the vocal tracts in humans (Rizzollatti \& Arbib, 1998; Arbib, 2002; Corballis, 2009, 2010). Researchers have also linked the incidence of population level right-handedness in humans and primates to the evolving culture of tool use that posed demands of manual dexterity both in tool production and manipulation (Corballis, 2009; Steele \& Uomini, 2009). However, some authors are still looking for alternative explanations for the shared neural substrates of tool and language functions and even their proximity on the evolutionary time-scale (Stout \& Chaminade, 2012). Finally, the mirror system hypothesis and its implications for co-evolution of language along with tool-use has been a topic of much speculation in the recent years and provides ample reason for researchers to engage with research both tool-use and language. Apart from a vast array of clinical and neuroimaging research the hemispheric dominance for tool use has also been 
examined in behavioral studies. Verma \& Brysbaert (2011) used the visual half-field (VHF) paradigm, in which stimuli are presented to the left and to the right of the fixation point and participants have to respond to the stimuli. The authors found that while there was no visual field difference for object recognition, a significant right visual field (RVF) advantage was observed for tools in the tool recognition task, in line with the left hemisphere dominance for tool processing. Garcea, Almeida \& Mahon (2012) used a lateralized masked priming paradigm to test for a visual half field asymmetry in tool processing. Using tools and animals as target stimuli and identical or scrambled versions of the targets as primes, they reported that there was a RVF advantage in priming effects for tool targets but not for animal targets.

Verma and Brysbaert (2011) argued that the strongest evidence for laterality of tool use in VHF studies is obtained when a tool recognition task is combined with an object recognition task. The prediction then is that a RVF advantage will be observed for tool recognition, together with no VHF advantage for object recognition. This pattern of results rules out the possibility that the RVF advantage for tool recognition is confounded by an uncontrolled variable (e.g., in the display of stimuli, in the participants' attention allocation, or in the fixation of the central stimulus). A problem for the approach, however, was that it was impossible to find matched pictures of tools, non-tool objects and non-objects from the same database. As a result, Verma \& Brysbaert (2011) had to compare pictures of tools vs. non-object tools from one source with pictures of objects vs. non-objects from another source. A similar problem was present in Garcea, Almedia, and 
Mahon (2012), who compared different sets of animals and tools as stimulus categories. Research would be more straightforward if the same pictures could be used in all comparisons, both as targets and primes.

To have access to better stimulus materials, we decided to compile a new set of stimuli, which contains matched triplets of tools, non-tool objects, and non-objects, so that research can examine object and tool recognition in the same study with the same materials. To test the validity of the new stimulus set, we tried to replicate the findings of our previous study (Verma \& Brysbaert, 2011). As in that study, we make use of two experiments. First, an Object Recognition VHF experiment will be run, in which participants are presented with pictures of objects and non-objects in the left and right visual half-field. The participants have to decide whether the designated picture displays an object or a non-object using bi-manual responses. Second, a Tool Recognition VHF experiment will be run, in which the same pictures of objects are presented with pictures of tools and participants have to decide whether the indicated picture represents a tool or not. The object recognition experiment acts as a control experiment for the tool recognition experiment. If no visual field difference is observed for the objects in the first experiment and a significant RVF advantage for the tools in the tool recognition experiment, then we can safely assume that the VHF advantage is due to tool-specific brain activity and not to a confounded variable. 


\section{Method}

\section{Stimuli}

We had three types of stimuli drawn by an artist: pictures of objects, non-objects, and tools. We explained that it was important to have perceptually similar items that were comparable in terms of overall shape, contour, luminance, and so on. All stimuli were digitally generated and were similar to the line drawings of the IPNP pictures database (Snodgrass \& Vanderwart, 1980). The object pictures consisted of line-drawings of familiar objects, such as: book, boot, maize, asparagus, carrot, palm etc. The tool pictures consisted of line-drawings of familiar hand-manipulable tools like: knife, hammer, comb, pliers, etc. The non-object pictures were made in such a way that they would match a pairing of an object and a tool in overall shape, size, etc. Specifically, all figures were sized 150 x 150 pixels and presented as bitmap images. The figures extended for 3 degrees of visual angle. Figure 1 shows three examples of each category.

Insert Figure 1 about here 
In all, we managed to have 38 triplets of matched pictures representing an object, a tool and a non-object (see the Appendix). All pictures were sized 150 x 150 pixels. To make sure that our stimuli were perceived by the participants in the way we intended them, we ran two rating studies using 6-point Likert scales: the Object Rating Scale (ORS) and the Tool Rating Scale (TRS). In the ORS, 20 participants rated the 'objectness' of the object and non-object stimuli. For each stimulus they indicated how certain they were that the stimulus represented an object; with $1=$ least certain and $6=$ most certain. In the TRS, 20 new participants rated the 'toolness' of the tool and object stimuli, indicating with a rating from 1 to 6 how certain they were that the stimulus in question represented a tool $(1=$ least certain, $6=$ most certain $)$.

Figure 2 shows the results of the rating studies. In the Object Rating Scale, 36 of the 38 non-object stimuli, scored between 1 to 3 points, while 2 scored marginally between 3 and 3.5, indicating that all non-object stimuli were perceived as non-objects by the participants. Furthermore, all of the 38 object stimuli scored between 4 to 6 points, indicating that they were easily distinguishable from the non-objects. In the Tool Rating Scale, 34 out of the 38 non-tool objects scored between 1 to 3 points, 4 objects scored between 3 and 3.5. On the other hand 30 of the 38 tool objects scored between 4 to 6 points, while four objects scored between 3 and 4 , and four less than 3 . The four pictures with tool scores lower than 3 were all musical instruments, namely a guitar, saxophone, 
harp, and pipe. The 4 other objects which were not clearly categorized were cigarettes, zipper, kettle and wristwatch. All scores can be found in the Appendix.

We decided to use all stimuli in the validation study, so that we had data about all of them. Of course, researchers are free to omit the less clear ones if they want to run a study without ambiguous stimuli.

Insert Figure 2 here.

\section{Participants}

A group of 39 undergraduate students from Ghent University took part in both the object decision experiment and the tool decision experiment. They were all right handed and had normal or corrected to normal vision. The study in total took slightly over one hour and participants were paid $12 €$ and.

\section{Procedure}

Participants were seated in front of a 17" computer screen at a distance of $80 \mathrm{~cm}$. At this distance, the pictures subtended a visual angle of 4 degrees and were presented 
laterally between 3-7 degrees from the fixation location. Before the start of each experiment the participants were familiarized with the pictures that would be presented. They were given a tachistoscopic presentation of the stimuli (with similar presentation times as in the real experiment) and asked to name the pictures. They were corrected if needed.

For the first experiment, the pictures of the objects and the non-objects were used and participants had to decide whether one of the two bilaterally presented stimuli was an object or a non-object. For the second experiment, the same pictures of the objects were combined with those of the tools, and the participants had to decide whether one of the two bilaterally presented stimuli was a tool or not.

On each trial participants were first presented with a blank screen for $1000 \mathrm{~ms}$, followed by a fixation cross (sized 1 degree of visual angle) at the center of the screen for $300 \mathrm{~ms}$. The fixation cross was replaced by a display which had a centrally presented arrow (sized 1 degree of visual angle) pointing to the left or to the right, along with two pictures, one in the left visual field (LVF) and one in RVF. The duration of the display was $200 \mathrm{~ms}$, based on the research of Walker \& McSorley (2006) showing that participants are unable to initiate an eye movement within $200 \mathrm{~ms}$ if they have to attend to a stimulus at the fixation location (the central arrow in our case). The stimuli presented in LVF and RVF could represent an object or a non-object (in Experiment 1), or a tool or a non-tool (in Experiment 2). The stimuli in the VHFs could belong to the same category (compatible) or a different category (incompatible). Participants were instructed to attend 
to the stimulus in the VHF to which the central arrow pointed, and to decide whether it represented an object/non-object (Experiment 1) or a tool/non-tool (Experiment 2). In case the stimulus represented an object (Experiment 1) or a tool (Experiment 2), the participant had to press two buttons with the index fingers of both hands; otherwise they had to press buttons with the middle fingers of both hands. We used bi-manual responses to avoid a stimulus-response compatibility effect (i.e., responses by the right hand are faster to stimuli in RVF and vice-a-versa). Reaction Times and Accuracy were calculated based on the first key-press registered. Reaction time measurement started from stimulus offset, like in Verma and Brysbaert (2011). This means that $200 \mathrm{~ms}$ must be added to get the total processing time.

Depending on the VHF of stimulus presentation (LVF or RVF), the stimulus (object/non-object, tool/non-tool), and whether the stimulus in the contra-lateral visual field was from the same category (compatible vs. incompatible), eight types of trials could be formed. There were 80 instances of each type in each experiment, giving 640 trials in all. All participants started with the object vs. non-object decision task, and ended with the tool vs. non-tool task.

\section{Results}

The findings of the two experiments are summarized in Figures 3 and 4.

Insert Figures 3 and 4 here 
We ran a $2 \times 2 \times 2 \times 2$ omnibus ANOVA to compare the two tasks, having Task (Object recognition vs. Tool recognition), VHF (LVF vs. RVF), Response (yes vs. no), and Compatibility of the distractor in the opposite VHF (from the same vs. different category) as repeated measures. For the RT data, we obtained significant main effects of VHF $(\mathrm{LVF}=412.7 \mathrm{~ms}$ vs. $\mathrm{RVF}=406.7 \mathrm{~ms} ; \mathrm{F}(1,38)=10.09, \mathrm{p}<0.01)$, Response $(\mathrm{Yes}=$ 385.7 vs. $\mathrm{No}=433.7, \mathrm{~F}(1,38)=123.75, \mathrm{p}<0.01)$, and Compatibility (Incompatible $=$ 419.8 vs. Compatible $=399.6, \mathrm{~F}(1,38)=85.57, \mathrm{p}<0.01)$. The main effect of Task was not significant $($ Object Recognition $=405.1$ vs. Tool Recognition $=414.3, \mathrm{~F}(1,28)=0.24$, $\mathrm{p}>0.05)$.

Significant interaction effects were: Task x VHF $(F(1,38)=27.33, \mathrm{p}<0.01)$; Task $x$ Response $(F(1,38)=5.87, p<0.05)$; VHF $x$ Response $(F(1,38)=11.74, p<0.01)$; Task x VHF x Compatibility $(\mathrm{F}(1,38)=4.96, \mathrm{p}<0.05)$; Response $x$ Compatibility $(\mathrm{F}(1,38)=$ 91.93, $\mathrm{p}<0.01)$ and Task $x$ Response $x$ Compatibility $(F(1,38)=30.33, p<0.01)$.

We also calculated the same omnibus ANOVA on percentage accuracy. This revealed significant a main effect of Compatibility (Incompatible $=87.3 \%$ correct vs . Compatible $=89.9 \%, \mathrm{~F}(1,38)=50.86, \mathrm{p}<0.01)$. The effect of Task was close to significance $($ Object Recognition $=89.9$ vs. Tool Recognition $=87.2, \mathrm{~F}(1,38)=4.03, \mathrm{p}$ $=0.052)$. The significant interaction effects were: Task x VHF $(F(1,38)=4.57, p<0.05)$, VHF x Compatibility $(\mathrm{F}(1,38)=6.09, \mathrm{p}<0.05)$ and Response $x$ Compatibility $(\mathrm{F}(1,38)$ 
$=16.30, \mathrm{p}<0.01)$. The interpretation of these effects will be clearer when we have a look at the ANOVAs for the two tasks separately.

We ran a 2 × 2 × 2 ANOVA for each task, i.e. Object Recognition and Tool Recognition, to understand the effects of VHF, Response and Compatibility better. In the Object Recognition task, the 2 × 2 × 2 ANOVA on RTs revealed a significant main effect of Response (Yes i.e. Objects $=376.7 \mathrm{~ms}$ vs. No i.e. Non-objects $=433.6$ ) and Compatibility (Incompatible $=415.9$ vs. Compatible $=394.4$ ). The main effect of VHF was not significant $(\mathrm{LVF}=403.4$ vs. $\mathrm{RVF}=406.8, \mathrm{~F}(1,38)=1.88, \mathrm{p}>0.05)$. The significant interactions were $\operatorname{VHF} x$ Response $(F(1,38)=5.44, p<0.05)$ and Response $x$ Compatibility $(\mathrm{F}(1,38)=110.07, \mathrm{p}<0.01)$. The interaction between VHF and Response seems to be driven mainly by the no responses (for non-objects): There was no significant difference in RTs between LVF and RVF for objects $(\mathrm{LVF}=377.2 \mathrm{vs.} \mathrm{RVF}=376.2, \mathrm{~F}(1$, 38) $=\mathrm{F}(1,38)=0.088, \mathrm{p}>0.05)$ while there was a significant LVF advantage in RTs for non-objects $(\mathrm{LVF}=429.6, \mathrm{RVF}=437.5, \mathrm{~F}(1,38)=9.13, \mathrm{p}<0.01)$. The interaction between response $\mathrm{x}$ compatibility was also mainly driven by the effect of compatibility for non-objects; non-objects seemed to become particularly salient in case of compatible information across the visual fields and elicit faster RTs as compared to when stimuli in the two visual fields were from different categories (Incompatible $=456.2$, Compatible $=$ 410.9, $\mathrm{F}(1,38)=130.96, \mathrm{p}<0.01)$. For the Accuracy data, the $2 \times 2 \times 2$ ANOVA revealed a significant main effect of compatibility (Incompatible $=88.7$, Compatible $=$ 91.2, $\mathrm{F}(1,38)=20.11, \mathrm{p}<0.01)$. The interaction effects of VHF x Compatibility $(\mathrm{F}(1$, 
$38)=4.23, \mathrm{p}<0.05)$ and Response $\mathrm{x}$ Compatibility $(\mathrm{F}(1,38)=14.48, \mathrm{p}<0.01)$ were significant, indicating that overall participants were more accurate in cases where information across the two visual fields belonged to the same category. The three way interaction between VHF, Response and Compatibility was not significant $(\mathrm{F}(1,38)=$ $0.147, \mathrm{p}>0.05)$

In the Tool Recognition task, the $2 \times 2 \times 2$ ANOVA for RTs revealed significant main effects of VHF $(\mathrm{LVF}=421.9 \mathrm{~ms}, \mathrm{RVF}=406.6 \mathrm{~ms},(\mathrm{~F}(1,38)=32.74, \mathrm{p}<0.01)$, Response $($ yes $=394.8$, no $=433.8, F(1,38)=53.27, \mathrm{p}<0.01)$ and Compatibility $($ Incompatible $=423.7$, Compatible $=404.8, \mathrm{~F}(1,38)=58.32, \mathrm{p}<0.01)$. The significant interaction effects were VHF x Compatibility $(F(1,38)=5.65, \mathrm{p}<0.05)$ and Response $\mathrm{x}$ Compatibility $(\mathrm{F}(1,38)=21.79, \mathrm{p}<0.01)$. The interaction between VHF and Response failed to reach significance $(\mathrm{F}(1,38)=2.57, \mathrm{p}>0.05)$. In the tool recognition task, participants responded significantly faster to pictures of tools presented in RVF than in $\mathrm{LVF}(\mathrm{LVF}=404.6, \mathrm{RVF}=384.9, \mathrm{~F}(1,38)=20.05, \mathrm{p}<0.01)$. Also participants responded significantly faster to tools in the compatible condition than in the incompatible condition (incompatible $=399.5$, compatible $=390.1, \mathrm{~F}(1,38)=8.51$, $\mathrm{p}<0.01)$. For the non-tool objects, participants were also significantly faster in RVF (LVF $=439.2, \mathrm{RVF}=428.4, \mathrm{~F}(1,38)=11.43, \mathrm{p}<0.01)$; also similar to the tools, non-tool objects were responded to significantly faster in the compatible condition (incompatible $=$ 448.1$, compatible $=419.5, \mathrm{~F}(1,38)=78.08, \mathrm{p}<0.01)$. For the Accuracy data, the $2 \times 2 \mathrm{x}$ 2 ANOVA revealed significant main effects of $\operatorname{VHF}(\mathrm{LVF}=86.7, \mathrm{RVF}=87.8, \mathrm{~F}(1,38)$ 
$=4.26, \mathrm{p}<0.05)$ and compatibility (incompatible $=85.8$, compatible $=88.6, \mathrm{~F}(1,38)=$ $30.36, \mathrm{p}<0.01)$.

To be sure that the inclusion of the 8 tool stimuli, which did not score above 4 in the Tool Rating Scale, did not distort the findings; we reanalyzed the data from the tool recognition study excluding these stimuli. Fortunately, the results did not change at all. There was again a main effect of VHF ( $\mathrm{LVF}=420.9 \mathrm{~ms}$ vs. $\mathrm{RVF}=406.1 \mathrm{~ms}), F(1,38)=$ $35.30, \mathrm{p}<.01$, Response (yes $=393.2 \mathrm{~ms}$ vs. no $=433.8 \mathrm{~ms}$ ), $F(1,38)=53.48, \mathrm{p}<.01$, and Compatibility $($ compatibility $=403.6 \mathrm{~ms}$ vs. incompatibility $=423.4 \mathrm{~ms}), F(1,38)=$ 60.24. The interaction between VHF x Compatibility, F $(1,38)=7.15, \mathrm{p}<0.05(\mathrm{p}=$ $0.011)$ and between Response $\mathrm{x}$ Compatibility was also again significant, $\mathrm{F}(1,38)=$ $21.53, \mathrm{p}<0.01$. Finally, the VHF advantage for tools did not change either $(\mathrm{LVF}=412.3$ ms vs. $\mathrm{RVF}=385.3 \mathrm{~ms}), F(1,38)=25.24, \mathrm{p}<0.01$. As the initial analysis already excluded the incorrect trials; the exclusion of the stimuli did not affect the percentage accuracy pattern.

All in all, the addition of the less clear stimuli did not make a difference in the pattern of results; and probably they can be kept included in the stimuli pool.

\section{Discussion}

To improve research on tool recognition (in particular, laterality research), we developed a set of pictures in which tools, non-tool objects, and non-objects were made 
as similar as possible (see Figure 1 for examples; see the Appendix for a list of all the picture names; see the supplementary materials for files of the pictures). To validate the new stimulus materials, we used them in the two experiments described by Verma and Brysbaert (2011): An object and a tool recognition experiment. On the basis of our previous results (and in line with the existing literature), we expected no VHF difference for the object-recognition experiments, whereas a significant RVF advantage was predicted for the tool recognition experiment.

We indeed obtained a very robust interaction between Task (object recognition vs. tool recognition) and VHF. The results of the Object Recognition experiment pointed to equal performance in LVF and RVF for responses to objects, combined with an $8 \mathrm{~ms}$ LVF advantage for responses to non-objects. The latter was also observed in Verma and Brysbaert (2011) and is not contradictory to the expectations. Non-objects are by definition unnamable, and arguably the participants resort to a spatial analysis for rejecting these stimuli as existing objects. Because spatial analysis predominantly relies on the right hemisphere, especially in the case of right handers (Vogel, Bowers \& Vogel, 2003), a LVF advantage is not surprising.

More importantly, we replicated the predicted RVF advantage for tool recognition. This was true for the yes-responses to tools (20 ms RVF advantage) and for the noresponses to the non-tool objects (11 ms RVF advantage). In Verma \& Brysbaert (2011) the differences were respectively $17 \mathrm{~ms}$ and $3 \mathrm{~ms}$. The RVF advantage becomes even larger if the analysis is limited to the incompatible trials, when the distractor in the 
opposite VFH was not from the same category as the target. Then the difference increases to $26 \mathrm{~ms}$ for the tools and to $15 \mathrm{~ms}$ for the non-tool objects.

Also interesting is the finding that overall response times and accuracies were very similar in both tasks $(\mathrm{RT} \approx 410 \mathrm{~ms}, \%$ correct $\approx 89 \%)$. This makes it easier to interpret the interaction (Loftus, 1978) and is much better than in Verma \& Brysbaert (2011), where the object recognition task $(451 \mathrm{~ms})$ was significantly faster than the tool recognition task $(474 \mathrm{~ms})$ and also more accurate $(85.2 \%$ vs. $84.2 \%$ correct). This illustrates the importance of using the same, controlled stimuli in both tasks.

To encourage more researchers to investigate tool and object recognition, we make the new set of stimuli available as supplementary materials. The main omission of the Snodgrass \& Vanderwart (1980) stimulus bank is that it does not have pictures of nonobjects. This makes it impossible for researchers to run object vs. non-object discrimination tasks on these stimuli. In addition, we found it hard to compile enough well-matched stimulus pairs of tools and non-tool objects with this stimulus bank. Other pictures databases (e.g., van Diepen \& De Graaf, 1994) do contain pictures of nonobjects, but have a shortage of tool pictures. Because the drawing style of both datasets is different, it is impossible to combine a picture from one set with that of another set, without introducing all types of low-level visual confounds (e.g., the thickness of the lines or the level of texture in the picture). Now, we have a set of stimulus materials addressing these concerns. This should encourage further research into tool use and the lateralization of the skills involved. 


\section{References}

Alcock, J. (1972). The evolution of the use of tools by feeding animals. Evolution, 26, 464-473.

Amborse, S.H. (2001). Paleolithic technology a human evolution. Science, 291, 1748-1753.

Beck, B. B. (1980). Animal Tool Behavior: the Use and Manufacture of Tools. New York: Garland STPM Press.

Beauchamp, M., Lee, K., Haxby, J., \& Martin, A. (2002). Parallel visual motion processing streams for manipulable objects and human movements. Neuron, 34, 149-159.

Beauchamp, M.S. and Martin, A. (2007). Grouding Object Concepts in Perception and action: Evidence from fMRI studies of tools. Cortex, 43, 461-468.

Boesch, C. and Boesch, H. (1990). Tool use and tool making in wild chimpanzees. Folia Primatologica, 54, 86-99.

Buxbaum, L. J., Schwartz, M. F., \& Carew, T. G. (1997). The role of semantic memory

Buxbaum, L. J., Veramonti, T., \& Schwartz, M. F. (2000). Function and manipulation

By Capuchin Monkeys (Cebus apella). Journal of Comparative Psychology, 101(2), 159-168.

Chao, L. L., \& Martin, A. (2000). Representation of manipulable man-made objects in the dorsal stream. Neuroimage, 12, 478-484.

Chao, L.L, Haxby, J. V., and Martin, A. (1999). Attribute-based neural substrates in temporal cortex for perceiving and knowing about objects. Nature Neuroscience, 10 (2), 913-919. 
Choi, S. H., Na, D. L., Kang, E., Lee, K. M., Lee, S. W., \& Na, D. G. (2001). Functional magnetic resonance imaging during pantomiming tool-use gestures. Experimental Brain Research, 139, 311-317.

Frey, S. H. (2008). Tool use, communicative gesture and cerebral asymmetries in the modern human brain. Philosophical Transactions of the Royal Society of London Series B, 363, 1951-1957.

Frey, S.H. (2004). The neural bases of complex tool use in humans. Trends in Cognitive Sciences, 8(2), 71-78.

Frey, S.H. (2007). What puts the how in where? Tool use and the divided visual streams hypothesis. Cortex, 43, 368-375.

Frey, S.H., Funnell, M.G., Gerry, V. E., and Gazzaniga, M. S. (2005). A dissociation between the representation of tool-use skills and hand dominance: Insights from Left and Right Handed Callosotomy Patients. Journal of Cognitive Neuroscience, 17(2), 262-272.

Frey, S.H., Newman-Norlund and Grafton, S.T. (2005). A Distributed Left Hemisphere Network Active During Planning of Everyday Tool Use Skills. Cerebral Cortex, 15, 681-695.

Fridman, E.A., Immisch, I., Hanakawa, T., Bohlhalter, S., Waldvogel, D., Kansaku, K., Wheaton, L., Wu, T., and Hallett, M. (2006). The role of the dorsal stream for gesture production. Neuroimage, 29, 417-428.

Garcea, F. E., Almeida, J., and Mahon, B.Z. (2012). A right visual field advantage for visual processing of manipulable objects. Cognitive Affective Behavioral Neuroscience, doi 10.3758/s13415-012-0106-x

Goldenberg, G., \& Spatt, J. (2009). The neural basis of tool use. Brain, 132, 1645-1655.

Goldenberg, G., Hermsdorfer, J., Glindemann, R., Rorden, C., and Karnath, H. O. (2007). Pantomime of Tool Use Depends on Integrity of Left Inferior Frontal Cortex. Cerebral Cortex, 17, 2769-2776.

Grezes, J., \& Decety, J. (2001). Does visual perception of objects afford action? Evidence from a neuroimaging study. Neuropsychologia, 40, 212-222.

Hart, B. L., Hart, L. A., McCoy, M. and Sarath, C. R. (2001). Cognitive behaviour in asian elephants: Use and modification of branches for fly switching. Animal Behaviour, 62, 839-847.

Hermsdorfer, J., Li, Y., Randerath, J., Goldenberg, G., and Johanssen, L. (2012). Tool use without a tool: kinematic characteristics of pantomiming as compared to actual use and the effect of brain damage. Experimental Brain Research, 218, 201-214. 
Hunt, G. R. (1996). Manufacture and use of hook-tools by new Caledonian crows. Nature, 379, 249-251.

in object use. CognitiveNeuropsychology, 14, 219-254.

Kallenbach, M. L., Brett, M., \& Patterson, K. (2003). Actions speak louder than functions: The importance of manipulability and action in tool representation. Journal of Cognitive Neuroscience, 15(1), 30-46.

Kroliczak, G. and Frey, S.H. (2009). A common network in the Left Cerebral Hemisphere Represents Planning of Tool Use Pantomimes and Familiar Intransitive Gestures at the Hand - Independent Level. Cerebral Cortex, 19, 2396-2410.

Kru"tzen, M., Mann, J., Heithaus, M. R., Connor, R. C., Bejder, L. \& Sherwin, W. B. (2005). Cultural transmission of tool use in bottlenose dolphins. Proceedings of the National Academy of Sciences, U.S.A., 102, 8939-8943.

Lefebvre, L., Nicolakakis, N. and Boire, D. (2002) Tools and brains in birds. Behaviour, 139, 939-973.

Lewis, J. W. (2006). Cortical networks related to human use of tools. Neuroscientist, 12, 211231.

Loftus, G. R. (1978). On interpretations of interactions. Memory and Cognition, 6(3), 312-319.

Oakley, K.P. (1956) Man the tool-maker. London: British Museum.

Osiurak, F., Jarry, C., Allain, P., Aubin, Ghisliaine, Etcharry-Bouyx, F., et al. (2009). Unusual use of objects after unilateral brain damage. The technical reasoning model. Cortex, 45, 769-783.

Perani, D., Schnur, T., Tettamanti, M., Gorno Tempini, M., Cappa, S. F., \& Fazio, F. (1999). Word and picture matching: A PET study of semantic category effects. Neuropsychologia, 37, 293-306.

Preston, B. (1998). Cognition and tool use. Mind and Language, 13, 513-547.

Randerath, J., Goldenberg, G., Spijkers, W., Li, Y., \& Hermsdorfer, J. (2010). Different left brain regions are essential for grasping a tool compared with its subsequent use. NeuroImage, 53, 173-180.

Snodgrass, J. G., \& Vanderwart, M. (1980). A Standardized set of 260 pictures: Norms for name agreement, image agreement, familiarity and visual complexity. Journal of Experimental Psychology: Human Learning and Memory, 6(2), 174-215.

tool knowledge in apraxia: Knowing "what for" but not "how". Neurocase, 6, 83-97. 
van Diepen, P. M. J., \& De Graef, P. (1994). Line-drawing library and software toolbox (Psych. Rep. No. 165). In Laboratory of experimental psychology. Belgium: University of Leuven.

van Lawick-Goodall, J. (1970). Tool-using in primates and other vertebrates. In: Advances in the Study of Behavior. Vol. 3 (Ed. By D. Lehrman, R. Hinde \& E. Shaw), pp. 195-249. New York: Academic Press.

Van Schaik, C.P., Ancrenaz, M., Borgen, G., Galdikas, B., Knott, C.D., Singleton, I., Suzuki, A., Utami, S.S. and Merrill, M. (2003). Orangutan Cultures and the Evolution of Material Culture. Science, 299, 102-105.

Verma, A. and Brysbaert, M. (2011). A right visual field advantage for tool-recognition in the visual half field paradigm. Neuropsychologia, 49, 2342-2348.

Vingerhoets, G., Alderweireldt, A.S., Vandemaele, P., Cai, Q., Van der Haegen, L., Brysbaert, M., and Achten, E. (in press). Praxis and language are linked: Evidence from colateralization in individuals with atypical language dominance.

Vogel, J. J., Bowers, C.A. and Vogel, D.S. (2003). Cerebral lateralization of spatial abilities; a meta-analysis. Brain and Cognition, 52, 197-204.

Westergaard, G. C. and Fragaszy, D. M. (1987). The Manufacture and Use of Tools by Capuchin monkeys (Cebus paella). Journal of Comparative Psychology, 101 (2), 159-168. 


\section{Figures:}
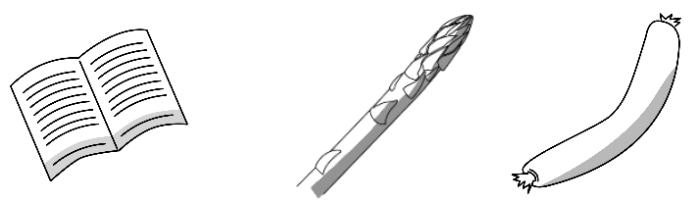

Objects
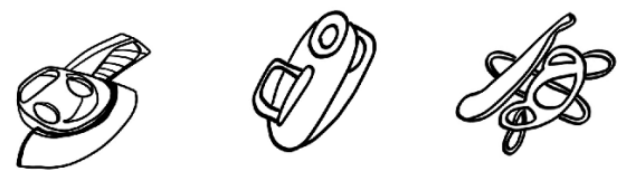

Non-Objects
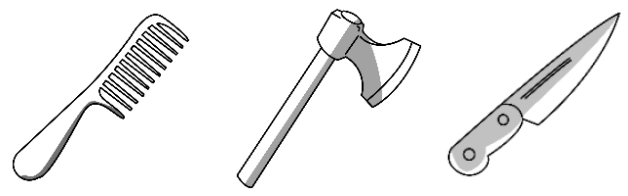

Tools

Figure 1: Showing examples of Objects (Non-tools), Non-Objects and Tools. While Objects and Non-Objects were used in Experiment 1, Objects and Tools were used in Experiment 2. 


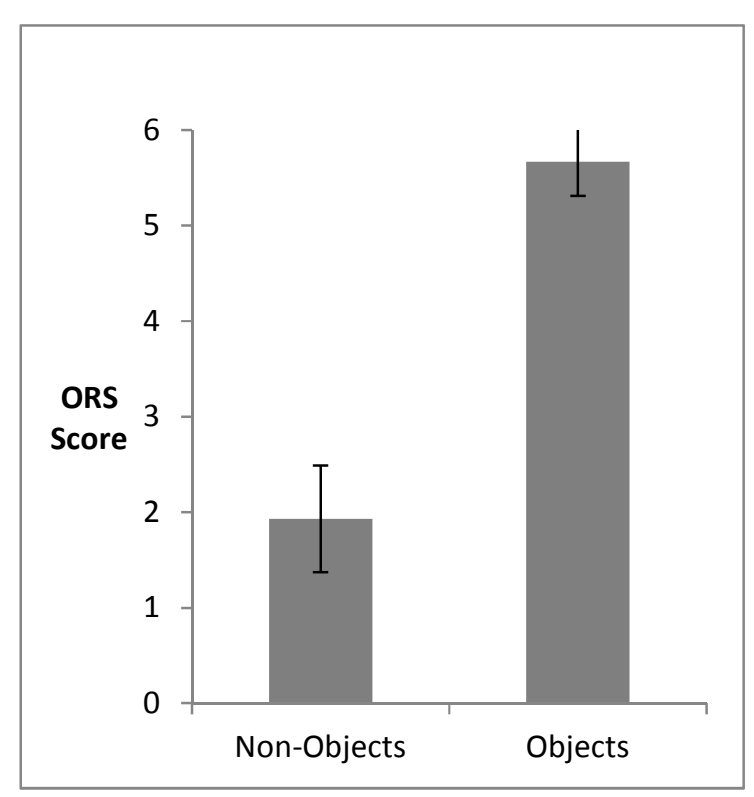

(A) Object Rating Scale

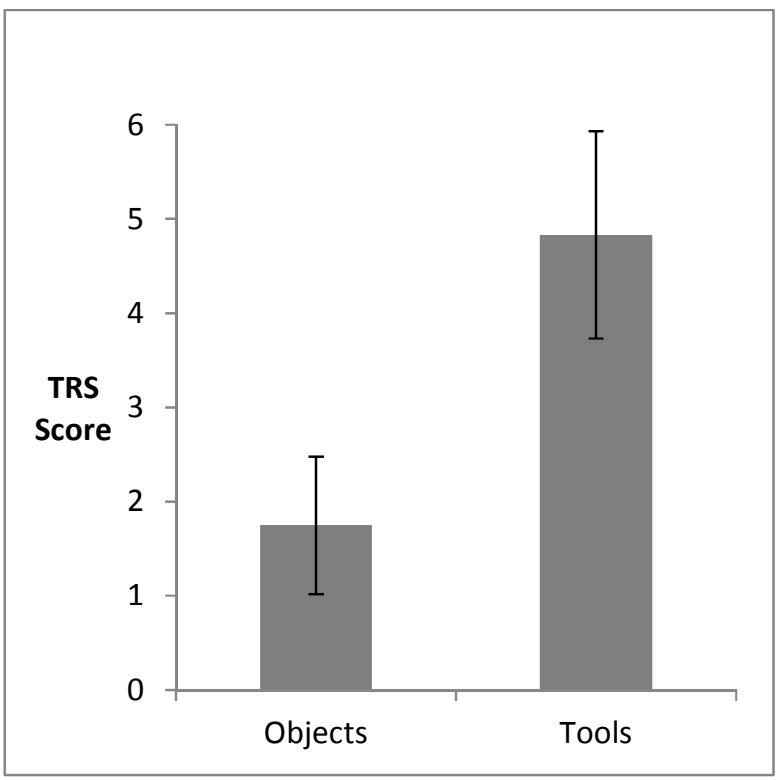

(B) Tool Rating Scale

Figure 2: Mean ratings of the pictures on the Object Rating Scale (ORS) and the Tool Rating Scale (TRS). In the object rating scale: a score of 1-3 corresponds to non-objects and a score of 3-6 corresponds to an object. In the tool rating scale: a score of 1-3 corresponds to an object and a score of 3-6 corresponds to a tool. See the Appendix for the rating values of the individual stimuli. 

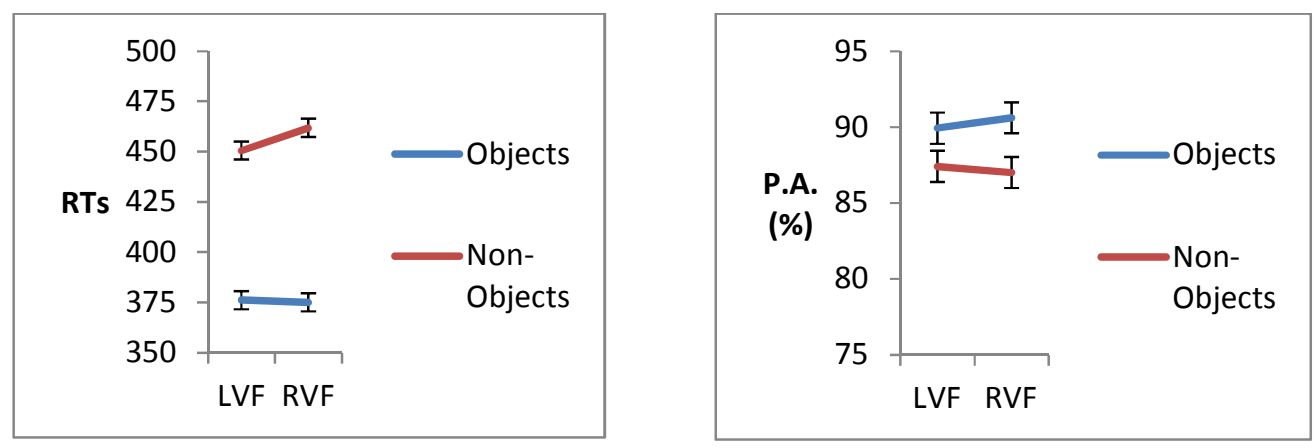

Figure 3: The results from the object recognition task, showing the lack of a clear VHF difference (except for the significant LVF advantage for non-objects in the RTs). Left panel: RT data; right panel: Percentage Acuuracy (P.A.) $\%$.
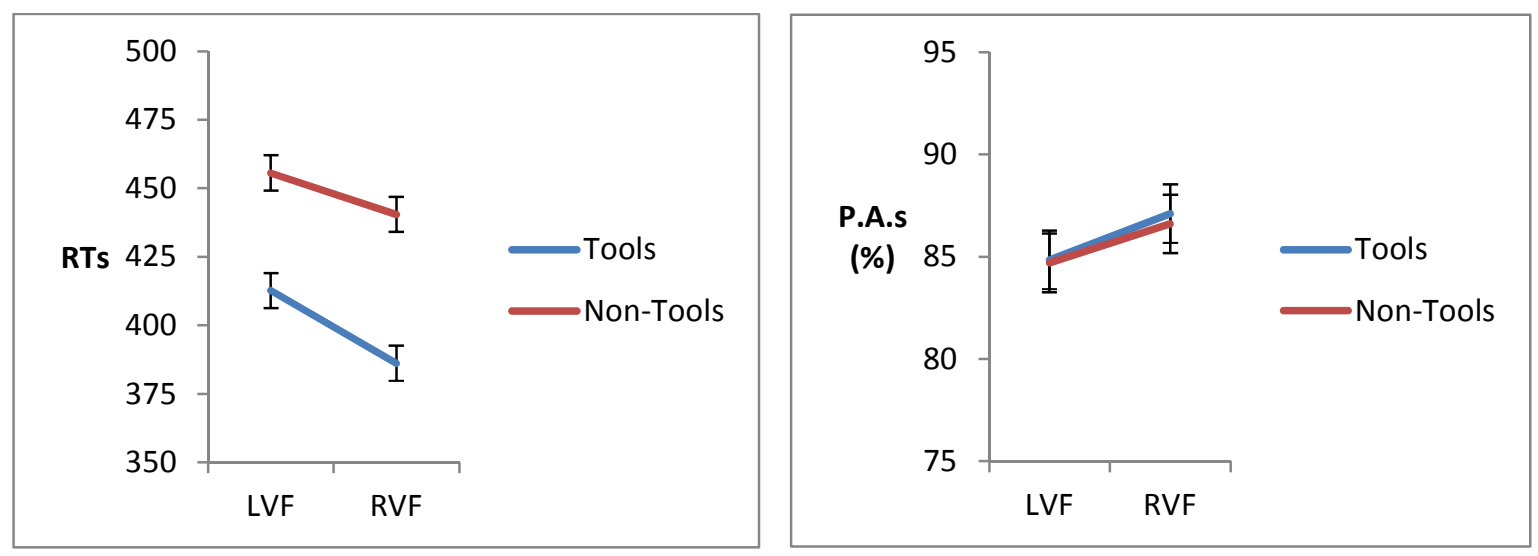

Figure 4: The results from the tool recognition task, showing the robust RVF advantage in the presence of a very similar performance level as in the object recognition task. Left panel: RT data; right panel: Percentage Accuracy (P.A.) \%. 
Appendix: Triplets of stimuli: objects, non-objects and tools, together with mean ratings in the Object Recognition Scale (ORS) and the Tool Recognition Scale (TRS).

Triplet 1

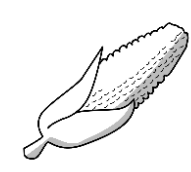

Maize

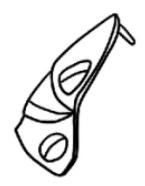

Non Object 1

$(\mathrm{ORS}=2.15)$

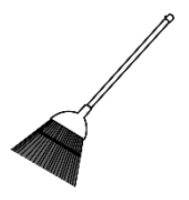

Broom

$(\mathrm{TRS}=5.35)$

Triplet 2

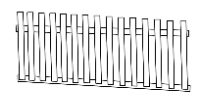

Fence

$(\mathrm{ORS}=5.5 ; \mathrm{TRS}=2.15)$

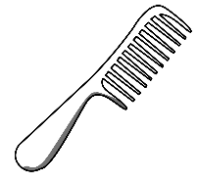

Comb

$(\mathrm{TRS}=5.0)$

Triplet 3

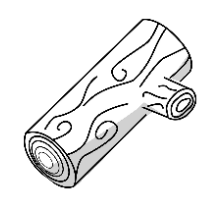

$\log$

$(\mathrm{ORS}=5.85 ; \mathrm{TRS}=1.65)$

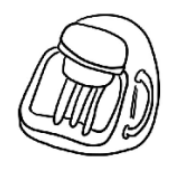

Non Object 2

$(\mathrm{ORS}=3.55)$
Non Object 3

$(\mathrm{ORS}=1.65)$

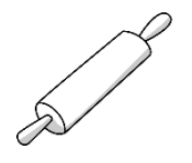

Rolling Pin

$(\mathrm{TRS}=5.3)$ 
Triplet 4

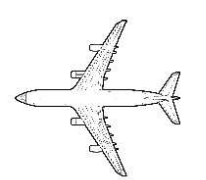

Airplane

$(\mathrm{ORS}=5.89 ; \mathrm{TRS}=2.5) \quad(\mathrm{ORS}=1.57)$

Triplet 5

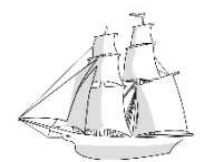

Ship

$(\mathrm{ORS}=5.84 ; \mathrm{TRS}=2.1)$

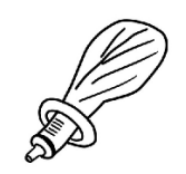

Non Object 5

$(\mathrm{ORS}=1.84)$

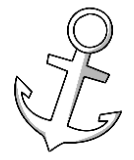

Anchor

$(\mathrm{TRS}=4.55)$

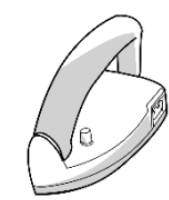

Iron

$(\mathrm{TRS}=5.35)$

\section{Triplet 6}

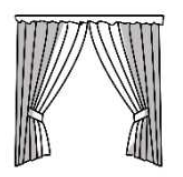

Curtain

$(\mathrm{ORS}=6.0 ; \mathrm{TRS}=1.55)$

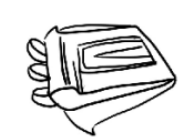

Non Object 6

$(\mathrm{ORS}=1.31)$

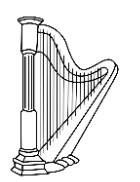

Harp

$(\mathrm{TRS}=2.6)$ 
Triplet 7

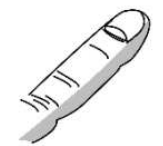

Finger

$(\mathrm{ORS}=5.89 ; \mathrm{TRS}=3.05)$

Triplet 8

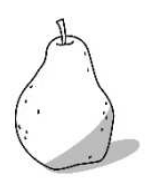

Pear

$(\mathrm{ORS}=5.94 ; \mathrm{TRS}=1.2) \quad(\mathrm{ORS}=1.84)$

Triplet 9

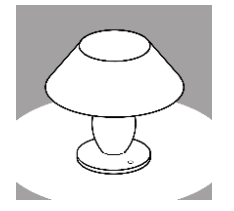

Lamp

$(\mathrm{ORS}=5.89 ; \mathrm{TRS}=2.0)$

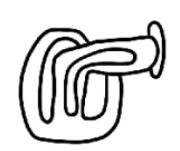

Non Object 7

$(\mathrm{ORS}=1.52)$

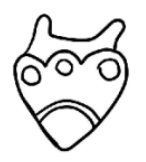

Non Object 8

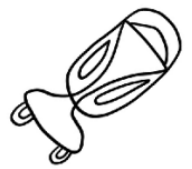

Non Object 9

$(\mathrm{ORS}=1.94)$

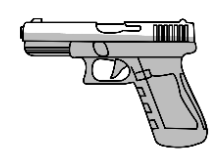

Pistol

$(\mathrm{TRS}=4.35)$

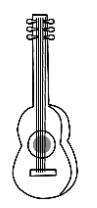

Guitar

$(\mathrm{TRS}=2.75)$

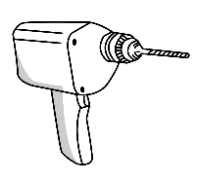

Hand-drill

$(\mathrm{TRS}=5.9)$ 
Triplet 10

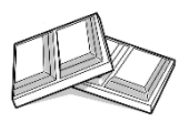

Chocolate

$(\mathrm{ORS}=5.42 ; \mathrm{TRS}=1.25)$

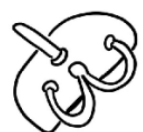

Non Object 10

$(\mathrm{ORS}=2.36)$

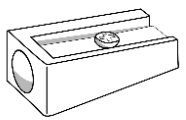

Sharpener

$(\mathrm{TRS}=5.4)$

Triplet 11

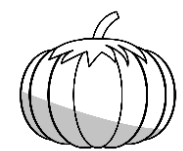

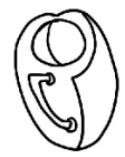

Non Object 11

$(\mathrm{ORS}=1.36)$

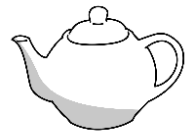

Kettle

$(\mathrm{TRS}=3.7)$

\section{Triplet 12}
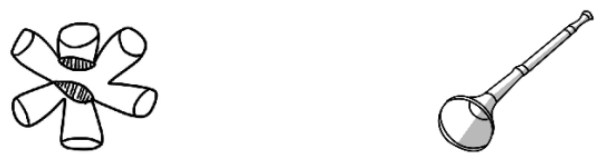

Bouquet

Non Object 12

Pipe

$(\mathrm{ORS}=5.73 ; \mathrm{TRS}=1.1)$

$(\mathrm{ORS}=1.68)$

$(\mathrm{TRS}=3.2)$ 
Triplet 13

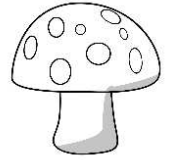

Mushroom

$(\mathrm{ORS}=5.84 ; \mathrm{TRS}=1.15)$

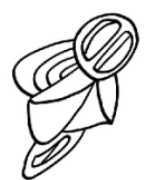

Non Object 13

$(\mathrm{ORS}=2.0)$

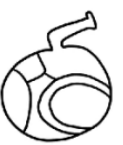

Non Object 14

$(\mathrm{ORS}=5.65 ; \mathrm{TRS}=3.35) \quad(\mathrm{ORS}=1.63)$

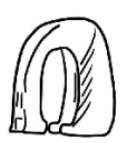

Non Object 15

$(\mathrm{ORS}=5.89 ; \mathrm{TRS}=1.1) \quad(\mathrm{ORS}=1.1)$

Triplet 15

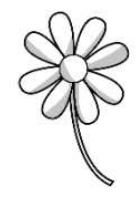

Flower

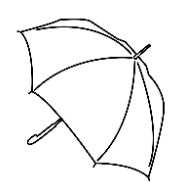

Umbrella

$(\mathrm{TRS}=4.2)$

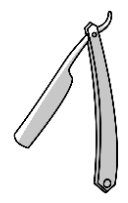

Razor

$(\mathrm{TRS}=5.2)$ 
Triplet 16

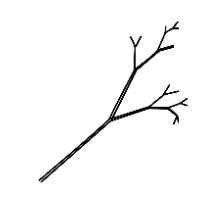

Twig

$(\mathrm{ORS}=5.84 ; \mathrm{TRS}=1.7)$

Triplet 17

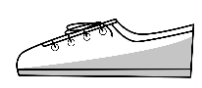

Shoe

$(\mathrm{ORS}=5.94 ; \mathrm{TRS}=2.15)$

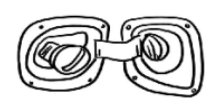

Non Object 16

$(\mathrm{ORS}=1.26)$

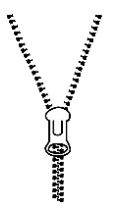

Zipper

$(\mathrm{TRS}=3.4)$

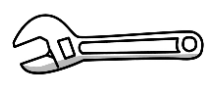

Wrench

$(\mathrm{TRS}=6.0)$

Triplet 18
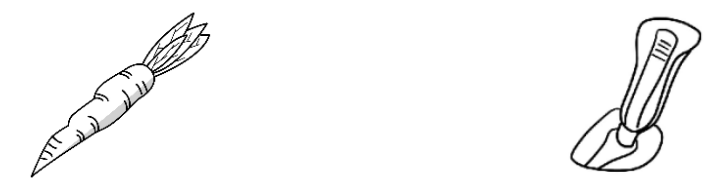

Non Object 18

$(\mathrm{ORS}=5.68 ; \mathrm{TRS}=1.05) \quad(\mathrm{ORS}=1.78)$

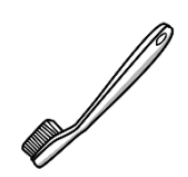

Toothbrush

$(\mathrm{TRS}=5.2)$

Triplet 19

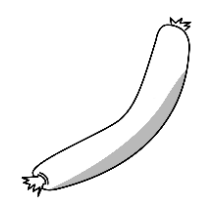

Sausage

$(\mathrm{ORS}=4.73 ; \mathrm{TRS}=1.1)$

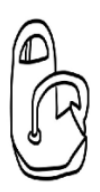

Non Object 19

$(\mathrm{ORS}=1.52)$

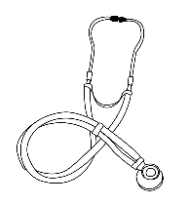

Stethoscope

$(\mathrm{TRS}=5.2)$ 
Triplet 20

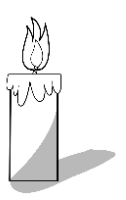

Candle

$(\mathrm{ORS}=5.84 ; \mathrm{TRS}=3.15)$

Triplet 21

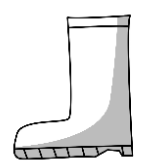

Boot

$(\mathrm{ORS}=5.57 ; \mathrm{TRS}=2.3)$

Triplet 22

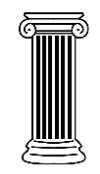

Pillar

$(\mathrm{ORS}=5.68 ; \mathrm{TRS}=1.9)$

Triplet 23

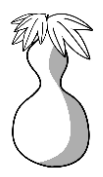

Bottle-gourd

$(\mathrm{ORS}=4.0 ; \mathrm{TRS}=1.1)$

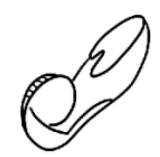

Non Object 20

$(\mathrm{ORS}=2.26)$

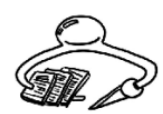

Non Object 21

$(\mathrm{ORS}=1.78)$

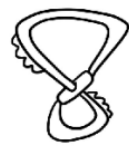

Non Object 22

$(\mathrm{ORS}=1.68)$

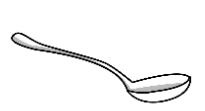

Spoon

$(\mathrm{TRS}=5.25)$

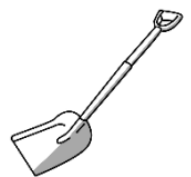

Shovel

$(\mathrm{TRS}=5.6)$

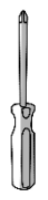

Screwdriver

$(\mathrm{TRS}=5.9)$

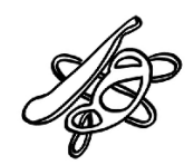

Non Object 23

$(\mathrm{ORS}=1.63)$

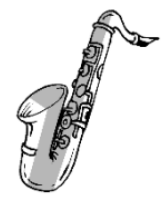

Saxophone

$(\mathrm{TRS}=3.0)$ 
Triplet 24

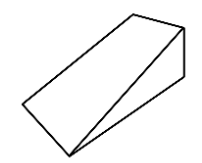

Incline

$(\mathrm{ORS}=5.26 ; \mathrm{TRS}=3.4)$

Triplet 25

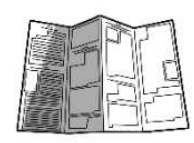

Pamphlet

$(\mathrm{ORS}=5.68 ; \mathrm{TRS}=2.9)$

Triplet 26

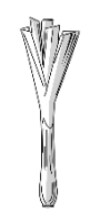

Spring-onion

$(\mathrm{ORS}=5.52 ; \mathrm{TRS}=1.15)$

Triplet 27

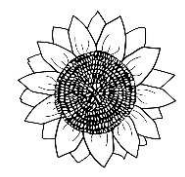

Sunflower

$(\mathrm{ORS}=5.63 ; \mathrm{TRS}=1.1)$

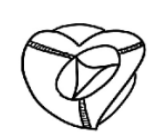

Non Object 24

$(\mathrm{ORS}=2.04)$

$(\mathrm{TRS}=6.0)$

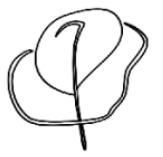

Non Object 25

$(\mathrm{ORS}=2.52)$

Ruler

$(\mathrm{TRS}=5.0)$

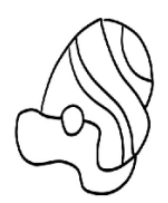

Non Object 26

$(\mathrm{ORS}=2.05)$

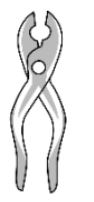

Pliers

$(\mathrm{TRS}=5.9)$

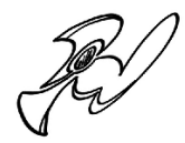

Non Object 27

$(\mathrm{ORS}=1.89)$

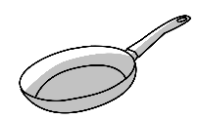

Pan

$(\mathrm{TRS}=4.65)$ 
Triplet 28

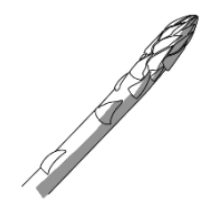

Asparagus

$(\mathrm{ORS}=5.47 ; \mathrm{TRS}=1.04)$

Triplet 29

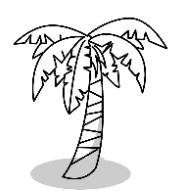

Palm

$(\mathrm{ORS}=5.73 ; \mathrm{TRS}=1.15)$

Triplet 30

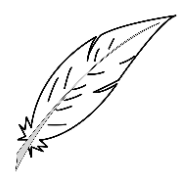

Feather

$(\mathrm{ORS}=5.78 ; \mathrm{TRS}=2.6)$

Triplet 31

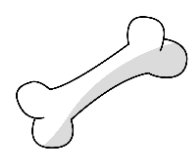

Bone

$(\mathrm{ORS}=5.78 ; \mathrm{TRS}=1.65)$

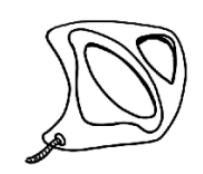

Non Object 28

$(\mathrm{ORS}=1.31)$

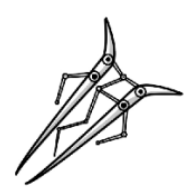

Non Object 29

$(\mathrm{ORS}=2.52)$

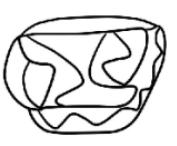

Non Object 30

$(\mathrm{ORS}=1.94)$

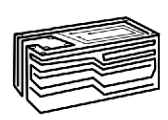

Non Object 31

$(\mathrm{ORS}=1.89)$

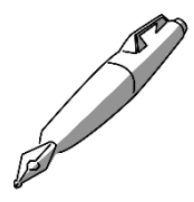

Pen

$(\mathrm{TRS}=5.05)$

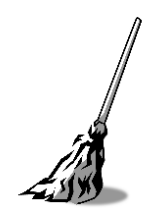

Mop

$(\mathrm{TRS}=5.6)$

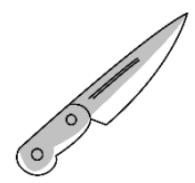

Knife

$(\mathrm{TRS}=5.8)$

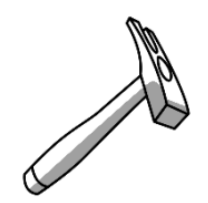

Hammer

$(\mathrm{TRS}=6.0)$ 
Triplet 32

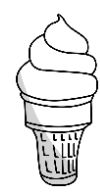

Ice-cream

$(\mathrm{ORS}=5.94 ; \mathrm{TRS}=1.1)$

Triplet 33

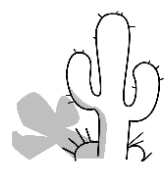

Cactus

$(\mathrm{ORS}=5.73 ; \mathrm{TRS}=1.1)$

Triplet 34

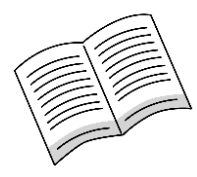

Book

(ORS 6.0; TRS = 1.85)

Triplet 35

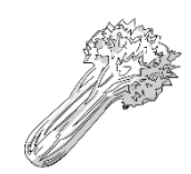

Celery

$(\mathrm{ORS}=5.42 ; \mathrm{TRS}=1.15)$

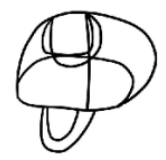

Non Object 32

$(\mathrm{ORS}=1.42)$

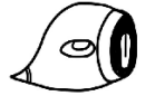

Non Object 33

$(\mathrm{ORS}=1.63)$

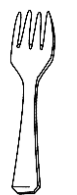

Fork

$(\mathrm{TRS}=5.35)$

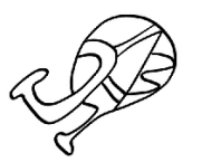

Non Object 34

$(\mathrm{ORS}=2.21)$

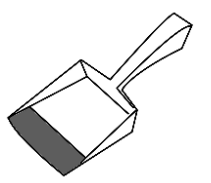

Dustpan

$(\mathrm{TRS}=5.35)$

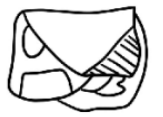

Non Object 35

$(\mathrm{ORS}=3.31)$

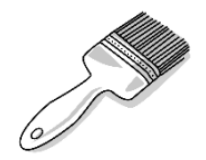

Paint-brush

$(\mathrm{TRS}=5.7)$ 
Triplet 36
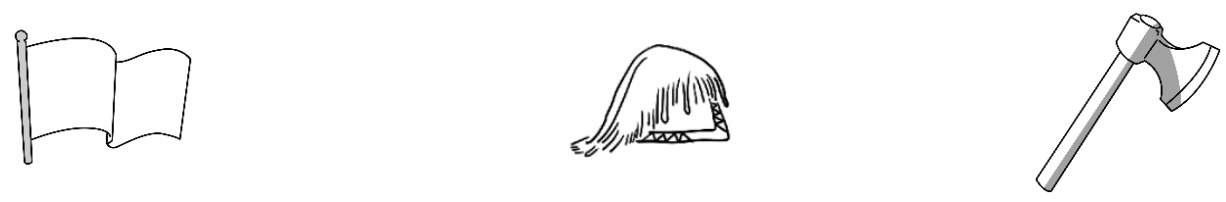

Flag

Non Object 36

Axe

$(\mathrm{ORS}=5.73 ; \mathrm{TRS}=2.35)$

$(\mathrm{ORS}=2.73)$

$(\mathrm{TRS}=5.85)$

Triplet 37
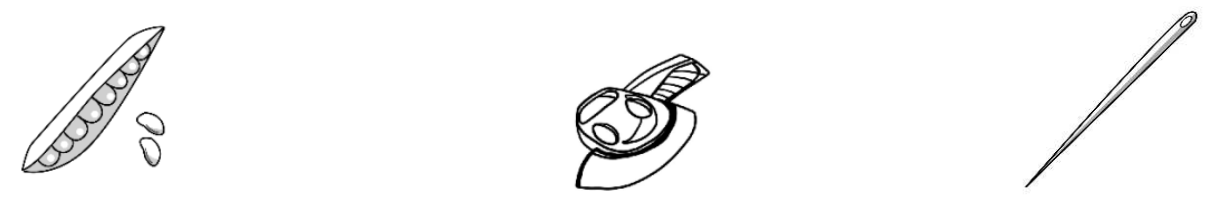

Beans

Non Object 37

Needle

$(\mathrm{ORS}=5.73 ; \mathrm{TRS}=1.15) \quad(\mathrm{ORS}=3.21)$

$(\mathrm{TRS}=4.85)$

Triplet 38
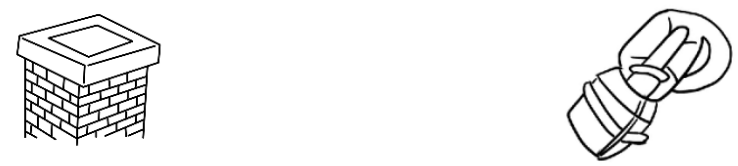

Chimney

Non Object 38

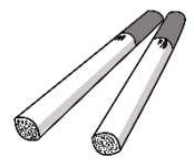

$(\mathrm{ORS}=5.89 ; \mathrm{TRS}=1.9)$

$(\mathrm{ORS}=1.42)$

Cigarettes

$(\mathrm{TRS}=1.55)$ 